\title{
Pour une nouvelle conceptualisation de la résistance à la marchandisation du carbone
}

\author{
Xavier P. Laberge \\ Université d'Ottawa
}

\section{Résumé}

La concentration de gaz à effet de serre dans l'atmosphère est alarmante et en guise de réponse, de plus en plus de gouvernements se tournent vers le marché afin de la réguler. Ce type de solution est privilégié pour son efficacité, sa flexibilité, la prévisibilité de ses résultats, les innovations technologiques qu'elle stimule et ses avantages politiques. Cependant, la marchandisation du carbone ne fait pas l'unanimité. Dans cette recherche, nous évaluons trois différentes perspectives d'analyse: néocoloniale, néogramscienne et polanyienne afin de répondre à la question suivante : quels sont les fondements théoriques de la résistance à la marchandisation du carbone? Cette analyse nous permet de comprendre les mouvements de contestation et de résistance aux nouveaux instruments économiques développés par le néolibéralisme dans l'objectif de diminuer l'amplitude des changements climatiques. Nous montrons qu'en plus de marchandiser la nature, le marché du carbone se trouve accusé par ces mouvements d'être à la fois néolibéral et néocolonial.

\section{Introduction}

La concentration de $\mathrm{CO}_{2}$ dans l'atmosphère est alarmante et n'a jamais été aussi élevée depuis au moins 420000 ans (Bachram: 5). En guise de réponse à cette crise, de plus en plus de gouvernements se tournent vers le marché afin de réguler les émissions de carbone $\left(\mathrm{CO}_{2}\right)($ Newell et Paterson $2010: 77)$. Ce type de solution est privilégié pour son efficacité, sa flexibilité, la prévisibilité de ses résultats, les innovations technologiques qu'elle stimule et ses avantages politiques (Baldwin 2008 : 262). Cependant, la marchandisation du carbone ne fait pas l'unanimité. Peter Newell et Matthew Paterson expliquent en effet que " comme avec chaque forme d'hégémonie, les marchés du carbone sont hautement contestés : plusieurs personnes s'opposent fondamentalement à l'idée de "marchandiser l'atmosphère" ॥ (Newell et Paterson 2010 : 77, traduction libre). Or, Lohmann explique que la contestation de ce mécanisme et ce qu'il nomme l' « overflow » ou débordement du système résultent des incertitudes reliées à cette solution (Lohmann, 2010 : 225)

La résistance au marché du carbone est maintenant en action et, même si ses différents mouvements ne partagent pas toujours les mêmes arguments ni les mêmes objectifs, elle influence la perception qu'ont les gouvernements ainsi que les citoyens et citoyennes du concept de marchandisation des émissions de gaz à effet de serre. Ironiquement, comme l'affirme Paterson, la résistance " construit le marché du carbone "(Paterson 2009: 252). Ce phénomène apparait contradictoire pour les organisations qui militent pour l'abolition des marchés du carbone plutôt que pour son optimisation. En effet, certaines organisations non gouvernementales (ONG) comme la WWF défendent un système de certifications pour le marché du carbone (WWF 2015) tandis que d'autres comme Carbon Trade Watch militent davantage pour son abolition (Carbon Trade Watch 2014).

La résistance à la marchandisation du carbone peut donc prendre plusieurs formes et les cadres d'analyse pour comprendre cette contestation sont aussi pluriels. Dans cette recherche, nous évaluerons trois différentes perspectives d'analyse. Nous croyons que chacune d'entre elles répond à certaines questions, 
mais qu'elles doivent être considérées conjointement afin de rendre une analyse substantielle des différents mouvements de contestation au marché du carbone.

Nous nous poserons la question suivante : quels sont les fondements théoriques de la résistance à la marchandisation du carbone? Pour y répondre, nous utiliserons les théories néocoloniale, néogramscienne ainsi que polanyienne. Nous croyons ainsi permettre l'approfondissement de la compréhension de cet enjeu primordial, car la contestation et sa réponse sont au cœur de la politique de l’ « économie carbonifère » (Newell et Paterson 2010 : 77).

\section{Le marché du carbone comme solution aux changements climatiques}

Les changements climatiques d'origine anthropique sont un des grands enjeux du $21^{\mathrm{e}}$ siècle et la gestion de ce problème est très complexe. Rappelons les bases physiques du phénomène : les bouleversements climatiques sont causés par l'accumulation d'émissions de gaz à effet de serre (GES) dans l'atmosphère et sont produits par les activités humaines sur Terre (GIEC 2014: 2). Cette accumulation crée un réchauffement planétaire par effet de serre. Cette dernière est occasionnée par la réflexion des rayons du soleil à l'intérieur de l'atmosphère par les GES, ce qui cause une accumulation de chaleur qui donne lieu à une augmentation de la température globale (GIEC 2007 : 79). Pour stopper cette réaction en chaîne, les émissions de GES doivent être réduites afin d'empêcher une augmentation de la fréquence des catastrophes climatiques, une augmentation du niveau des océans, l'élargissement des zones désertiques ainsi que plusieurs autres problèmes pouvant menacer des millions d'individus et engendrer la disparition d'une quantité importante de biodiversité (GIEC 2014 : 7-8). La limite de l'augmentation de la température moyenne mondiale a été fixée à deux degrés Celsius afin d'empêcher des bouleversements irréversibles d'une ampleur jugée dangereuse (Abbas 2010 : 19).

Or, l'économie actuelle est fondée sur la consommation d'énergie provenant principalement des carburants fossiles émetteurs de GES comme le pétrole et le charbon (GIEC 2014 : 5). Ainsi, plusieurs estiment que l'économie doit être " décarbonisée " afin d'éviter un emballement climatique extrême lié au dépassement de 1 à $2{ }^{\circ} \mathrm{C}$ par rapport aux niveaux de 1990 (GIEC 2007 : 19). Selon le Groupe d'experts intergouvernemental sur l'évolution du climat (GIEC), " les rejets annuels de dioxyde de carbone $\left(\mathrm{CO}_{2}\right)$ le plus important gaz à effet de serre anthropique - ont progressé de $80 \%$ environ entre 1970 et 2004 » (GIEC 2007 : 5) et ce, malgré l'adoption de la Convention-cadre des Nations unies sur les changements climatiques (CCNUCC) en 1992 ainsi que l'adoption subséquente du Protocole de Kyoto en 1997 (CCNUCC 2014b) et l'engagement de ses participants à lutter contre le réchauffement climatique mondial (CCNUCC 2014a).

En guise de solution, certains acteurs étatiques, mais aussi des entreprises et des acteurs financiers, ont suggéré d'utiliser le marché afin de réguler les émissions de carbone (Newell et Paterson 2010 : 77). En principe, donner une valeur, et donc un coût, au carbone à l'aide d'un mécanisme d'échange d'émissions ou encore en taxant tout simplement les émetteurs devrait être le moyen le plus efficace et le moins coûteux pour diminuer les émissions mondiales, mais les résultats sont pour l'instant encore incertains (GIEC 2014 : 30). Il existe déjà plusieurs marchés du carbone, dont ceux mis en place par le Protocole de Kyoto, soit le mécanisme de développement propre (MDP), l'application conjointe et le mécanisme d'échange volontaire, mais aussi le système communautaire européen d'échange de quotas d'émission (SCEQE ou EU ETS) et le marché du carbone australien (Stripple et Lövbrand 2010 : 167). Les marchés du carbone sont en expansions partout sur le globe avec des mises en œuvre actuelles ou futures en Californie, au Kazakhstan, en Chine, au Brésil, en Turquie et au Chili. Plusieurs considèrent aussi une intégration du REDD+ - mécanisme de réduction de la déforestation - dans un marché du carbone (Lane 
et Stephan $2015: 3$ ). Cela est sans compter les nombreux autres projets en préparation ailleurs dans le monde.

\section{Le marché du carbone : construction néolibérale}

Le marché du carbone est donc une des solutions les plus appréciées parmi les parties prenantes des négociations internationales sur les changements climatiques. Or, les résultats apparaissent faibles ou négligeables jusqu'à présent. Comment alors expliquer l'ampleur de l'engouement envers ce type de mécanisme? Selon Tamra Gilbertson et Oscar Reyes, le marché du carbone serait une manifestation de la domination de l'idéologie néolibérale au sein de l'économie mondiale (Gilbertson et Reyes 2009 : 18). En reposant sur le « pouvoir » du marché et son autorégulation, le marché du carbone apparaît donc comme une extension des politiques néolibérales (Newell et Paterson $2010: 82$ ).

Déjà, lors de l'élaboration du Protocole de Kyoto, l'idée des mécanismes de marché comme l'échange de droits d'émissions était majoritairement promue par les États-Unis et les environnementalistes promarchés (Lohmann 2006 : 49). L'Europe et les pays du Sud ont accepté ces mécanismes, car il ne semblait exister aucune autre alternative pour l'obtention d'un consensus mondial (Bedall 2013 : 204). Les groupes environnementaux ont aussi été convaincus par l'argument voulant que les mécanismes de marché fussent essentiels à l'adoption d'un traité sur les changements climatiques (Lohmann 2006 : 50).

Le capitalisme néolibéral a ainsi modelé la réponse au problème des changements climatiques par l'utilisation du marché, par la dominance de la finance, par l'accroissement des inégalités économiques mondiales et par sa fixation pour l'organisation en réseau (Newell et Paterson 2010: 83). La financiarisation du climat apparaît ainsi comme l'étape ultime de la conception néolibérale du marché du carbone par la gestion des droits d'émissions de la part des grandes institutions financières et la spéculation des permis par les fonds d'investissement (Lohmann 2009 : 32). La préférence des droits d'émissions sur la taxe provient donc en partie de l'intérêt du secteur dominant de la finance pour cette option (Newell et Paterson $2010: 85$ ).

Dans ce qui suit, nous présenterons trois cadres théoriques permettant la conceptualisation des mouvements de contestation et de résistance à la marchandisation du carbone. Cette résistance n'est pas un bloc monolithique et ses critiques ne le sont pas davantage. Il s'agira donc d'exposer trois parties de la contestation à l'aide de trois théories, soit le néocolonialisme, le néogramscisme et le polanyisme. Nous montrerons ensuite comment ces théories permettent d'expliquer la résistance aux marchés du carbone.

\section{Néocolonialisme}

L'internationalisation du concept de marché du carbone ne se fait pas sans problèmes. Une des principales résistances aux marchés du carbone provient des pays dits du "Sud » qui considèrent avoir été victimes à maints égards d'une nouvelle forme de colonialisme (Bachram 2004 : 10). Pour Heidi Bachram, il est même possible d'expliquer cette résistance par une opposition à un instrument ayant pour objectif de maintenir l'ordre international fondé sur le colonialisme. Elle nomme ce phénomène « carbon colonialism »(Bachram 2004 : 19).

En effet, le système d'échange de droits d'émission favorise inévitablement les parties qui possèdent les richesses au détriment de celles qui en ont moins (Baldwin 2008 : 267). Les différents participants étatiques, entrepreneuriaux ou individuels n'ont pas les mêmes possibilités au sein de ce marché. D'ailleurs, le système favorise la vente de droits d'émissions des participants les plus pauvres qui doivent 
plus tard racheter des droits à un prix plus élevé afin d'atteindre les objectifs de diminution (Page 2012 : 944). Le colonialisme du carbone devient flagrant lorsque des partisans du marché du carbone justifient la marchandisation de forêts indigènes au Sud afin de créer des puits de carbone (Lohmann $2008: 363$ ). Ceux-ci sont utilisés en vue de compenser des émissions industrielles de $\mathrm{CO}_{2}$ au nord. Le cœur de ce problème réside dans l'appropriation de terres servant à la monoculture ainsi que dans la plantation d'arbres servant de puits de carbone (Bachram 2004 : 11). Cela a pour conséquence d'appauvrir les communautés rurales dépendantes de ces territoires. En Ouganda, par exemple, des compagnies norvégiennes ont évincé 8000 personnes provenant de 13 villages par des cas de dépossession de terres (World Rainforest Movement 2000).

En ce qui a trait aux marchés du carbone volontaires, ils apparaissent comme un moyen de déculpabiliser les riches consommateurs du Nord qui peuvent continuer de maintenir leur niveau de vie en croyant régler la crise climatique par des investissements dans des projets réduisant en réalité de façon marginale, voire nulle, les émissions de GES (Paterson 2010 : 353). De plus, une des principales inquiétudes provient du renforcement des inégalités préexistantes par l'obtention des droits de polluer à des conditions plus favorables pour les riches que les utilisateurs pauvres en raison des possibilités d'information et d'arbitrage qui accompagnent leur richesse supérieure (Page 2012 : 944). En somme, les marchés du carbone semblent s'inscrire dans la poursuite d'une tradition coloniale bien établie entre pays du Nord et pays du Sud (Bachram 2004 : 20). L'explication des critiques néocoloniales à la marchandisation du carbone amène une compréhension accrue d'une partie de la résistance et de la contestation à celle-ci.

\section{Néogramscisme}

Antonio Gramsci a développé, pendant qu'il était en prison entre 1929 et 1935, plusieurs concepts permettant une meilleure compréhension de l'ordre internationale (Cox 2005[1993]) : 35). La théorie néogramscienne permet entre autres d'analyser les relations de pouvoir mondiales et plus particulièrement le pouvoir de la théorie, de l'idéologie et de la culture (O'Meara 2010 : 269). Le concept d'hégémonie de Gramsci montre que la suprématie d'un groupe social est fondée à la fois sur la coercition et le consentement (De Lucia 2009: 232). L'hégémonie est effective lorsque l'intérêt des dominants apparaît comme l'intérêt de la société en entier (Matt et Okereke 2015 : 115).

La structure de domination supportée par les classes dominantes de la société pour maintenir le système en place est nommée " bloc historique " (Cox 2005[1993]) : 39). Il y aurait alors convergence des intérêts des classes dominantes sur les aspects politique, économique, intellectuel et moral (De Lucia 2009 : 233). Ce bloc historique explique le consentement des masses par l'implication des intellectuels et de la société civile (De Lucia 2009 : 232). La communauté intellectuelle serait connectée de manière " organique " et supporterait la construction des « idéologies » et des institutions permettant la stabilité du bloc historique (Cox 2005[1993]) : 40-41). Vito De Lucia développe l'idée que le GIEC incarnerait cette intelligentsia organique qui, par sa composition issue très majoritairement des pays du Nord, reproduit son système de connaissances (De Lucia 2009 : 234).

La société civile joue, elle aussi, un rôle important dans le rapport de domination idéologique exprimée dans des " termes universalisant " permettant une apparence d'harmonie entre les classes dominantes et les classes subordonnées (Cox 2005[1993]) : 41). Par exemple, les ONG légitiment les organisations internationales hégémoniques et leurs idéologies en prenant part à leurs structures et en dialoguant avec elles (De Lucia 2009 : 234). Or, les organisations internationales "incarnent les règles qui facilitent l'expansion des ordres mondiaux hégémoniques; elles sont elles-mêmes le produit de l'ordre du monde hégémonique; légitiment idéologiquement les normes de l'ordre du monde; cooptent les élites des pays 
périphériques et absorbent les idées contre-hégémoniques » (Cox 2005[1993]) : 44, traduction libre). La CCNUCC, la Banque mondiale et le Fonds monétaire international (FMI) sont des organisations internationales qui, soit militent pour une économie capitaliste verte, soit appuient officiellement la création de marchés du carbone mondiaux. De son côté, la CCNUCC, par ses Conférences des parties (CdP) annuelles, permet et valorise la présence de la société civile et des ONG environnementales. Elle accrédite elle-même les personnes et organisations qui ont accès aux conférences. Non seulement cela valorise la présence des groupes et individus les plus aisés, mais il est possible de croire que cette institution favorise ceux et celles qui sont les plus proches des idéologies hégémoniques.

Dans le domaine économique, le bloc historique supporte définitivement l'idéologie néolibérale, ce qui a pour conséquence de favoriser la marchandisation du carbone comme solution à la problématique des changements climatiques (Matt et Okereke 2015 : 127). Par contre, il existe une résistance et une opposition à l'idéologie néolibérale hégémonique. Cette résistance est dite contre-hégémonique et, selon Gramsci, elle doit travailler à construire un "nouveau bloc historique " (Cox 2005[1993]) : 45). Selon Robert Cox, il est possible que les classes désavantagées et subordonnées s'allient afin de faire tomber le monopole libéral de l'ordre mondial (Cox 2005[1993]) : 46).

De son côté, Larry Lohmann décrit la résistance contre-hégémonique comme une lutte contre le néolibéralisme et la marchandisation de la terre (Lohmann 2012: 102). Si plusieurs ONG environnementales semblent avoir été cooptées par les organisations internationales et l'idéologie hégémonique (De Lucia 2009: 234-235), certaines continuent de s'inscrire dans un mouvement de contestation de l'ordre mondial actuel (Bedall 2013 : 209-213). Lohmann donne comme exemple des mouvements sociaux s'opposant à l'ordre néolibéral et sa gestion des problèmes climatiques : La Via Campesina, California Movement for Environmental Justice et les mouvements en Équateur, au Canada et au Nigéria s'opposant à l'extraction des combustibles fossiles (Lohmann 2012 : 102). Par conséquent, la théorie néo-gramscienne permet une conceptualisation d'une partie importante des mouvements de contestation et de résistance à la marchandisation du carbone.

\section{Polanyisme}

La résistance à la marchandisation du carbone peut aussi être expliquée en partie par les inquiétudes de la part de la société civile face au contrôle de la diminution des émissions de GES par un marché autorégulé. Karl Polanyi a nommé cette résistance le « contre-mouvement " (Polanyi 2014[1944] : 127). Ce contre-mouvement serait une " autodéfense " sociétale basée sur une marchandisation de produits fictifs (Lohmann 2010 : 225). En effet, pour Polanyi, il existe des marchandises créées pour le marché et ses échanges économiques, mais aussi des marchandises dites « fictives " telles que la terre, le travail et la monnaie (Polanyi 2014[1944] : 122-123). Ces " produits » peuvent être marchandisés et il est possible de leur assigner une valeur monétaire, même s'ils n'ont pas été conçus initialement pour la vente. Cette marchandisation de produits fictifs provoque de l'incertitude ou des "débordements " ainsi qu'une dynamique de résistance (Lohmann 2010 : 226). Pour Polanyi, il existe donc historiquement un double mouvement : un mouvement d'extension du marché et un contre-mouvement de résistance à l'extension du marché aux produits fictifs (Polanyi 2014[1944] : 127). Ainsi, ce contre-mouvement amène une régulation du marché par l'État et un frein à la marchandisation des produits fictifs.

Il devient donc possible d'exercer un parallèle avec la marchandisation de la pollution atmosphérique, du climat ou du carbone. Ces "produits " n'avaient certainement pas une vocation initiale de produit d'échange et peuvent donc être conçus comme des marchandises fictives telles que définies par Polanyi. Ces produits ont plutôt été intégrés au marché afin de poursuivre l'expansion capitaliste et tenter de 
répondre à la crise climatique à l'aide d'une solution néolibérale (Lohmann 2010 : 236). Cette solution a ouvert la porte à la financiarisation du carbone et a ainsi permis la spéculation et la participation des acteurs provenant du milieu de la finance, comme les banques et les compagnies d'investissement (Lohmann 2010 : 235-236). Les gouvernements élargiraient les marchés du carbone afin d'accroitre la liquidité, et ce, tout en favorisant une meilleure mesure des quantités de produits de carbone au lieu de travailler à une transition vers une économie moins dépendante aux énergies fossiles (Lohmann 2010 : 248).

La résistance peut dès lors être conceptualisée comme un contre-mouvement à la marchandisation du carbone et à l'incertitude qui en émerge. La principale inquiétude provient des potentielles bulles ou crises économiques que peut engendrer la spéculation déjà active au sein de certains marchés du carbone (Lohmann 2010 : 237). Une partie de la résistance peut être expliquée par un désir de certains membres de la société civile de prendre contrôle du marché " autorégulé " (Mittleman et Chin 1997 : 29). Les " débordements " permettent une critique des défauts et des problèmes reliés soit aux marchés du carbone, soit tout simplement à la marchandisation du carbone. Ainsi, le cadre théorique polanyien permet d'expliquer les revendications de groupes de pression et d'ONG contestant l'expansion du marché autorégulé, mais ne permet pas l'analyse des résistances non déclarées (Mittleman et Chin 1997 : 31). Le concept de marchandisation de produits fictifs explique une résistance au concept de marchandisation du carbone. Par exemple, le mouvement Climate Justice Now! avait fait une déclaration à Durban en 2004 dénonçant la marchandisation du carbone et avait fait un lien entre celle-ci et la marchandisation croissante de la terre, de la nourriture, du travail, des forêts, de l'eau, des gênes et des idées (Newell et Paterson 2010 : 89).

\section{Les mouvements sociaux de résistance aux marchés du carbone}

La résistance n'est pas formée d'un seul bloc, mais elle est plutôt constituée d'une multitude de résistances. Ces résistances peuvent diverger dans leur forme, par leur agent, dans leur localisation et dans leur échelle, et dans leurs stratégies (Mittleman et Chin 1997 : 34-35). Il est possible de croire que la résistance aux marchés du carbone est issue de mouvements sociaux et que ceux-ci peuvent être définis, selon Alberto Melucci, comme étant basés sur la solidarité, portant sur un conflit et contestant les limites du système dans lequel l'action se produit (Melucci 1985: 795). Nous retiendrons cette définition dans la présentation des différentes positions des mouvements sociaux contestant la marchandisation du carbone. II existe un large éventail de ces mouvements et, si certains visent l'amélioration des marchés du carbone, d'autres revendiquent leur abolition complète. Il est impossible dans le cadre de ce travail d'évaluer l'entièreté des mouvements de contestations. Cependant, nous tenterons de présenter un ensemble de groupes différents possédant des argumentaires variés et représentatifs de la contestation et de la résistance aux marchés du carbone.

L'Association pour la Taxation des Transactions financières et pour l'Action Citoyenne (ATTAC) est un groupe fondé en France en 1998 qui « se mobilise contre l'hégémonie de la finance et la marchandisation du monde » (ATTAC - France 2015 et ATTAC - Québec 2015). Par conséquent, c'est une organisation contre-hégémonique dans le sens néogramscien qui tente de contre-argumenter le discours hégémonique néolibéral. Comme son nom l'indique, ce groupe milite pour une réappropriation de l'économie par les citoyens et citoyennes, mais aussi pour leur participation accrue dans cette économie. Par exemple, ATTAC a publié en 2009 une brochure en vue des négociations de Copenhague défendant une justice climatique. Celle-ci contenait 16 propositions " orientées vers une mobilisation large des citoyens et citoyennes " (ATTAC-France 2009: 34-35). Dans ce document, ATTAC pourfend "le néolibéralisme et la globalisation économique » (ATTAC-France 2009 : 3), mais surtout l'utilisation du 
marché pour régler la crise climatique (ATTAC-France 2009 : 7). Il existe donc, en plus d'une perspective néogramscienne, un rapprochement avec l'argumentaire de Polanyi sur le marché. ATTAC soulève aussi l'aspect inéquitable de la répartition des projets du Mécanisme de développement propre (MDP) provenant de l'adoption du Protocole de Kyoto. Le MDP permet la compensation des émissions de $\mathrm{CO}_{2}$ dans les pays développés par des projets de réductions dans des pays en voie de développement (CCNUCC 2014c). L'organisme démontre qu'en 2008, des « 1243 projets [...] enregistrés dans 51 pays [...] [I]a majorité d'entre eux concerne l'Asie (66\%), ensuite l'Amérique latine (30\%) et enfin l'Afrique (3\%) " (ATTAC-France 2009 : 13).

À Copenhague, le 11 décembre 2009, un ensemble d'ONG environnementales se sont réunies au sommet alternatif Klimaforum09 pour rédiger la " Déclaration des peuples "; texte qui a été signé par plus de 300 organisations et finalement remis à la CCNUCC lors de la dernière journée de la $15^{e} \mathrm{CdP}$ (Klimaforum 2009). Dans ce texte, le " commerce du carbone et les mécanismes de compensation " sont présentés comme étant " des instruments faux et injustes puisqu'ils traitent le bien commun planétaire l'atmosphère - comme un produit qui peut être possédé et échangé " (Déclaration des peuples $2009: 8$ ). Le regroupement dénonce les injustices climatiques et les solutions basées sur l'économie de marché et promeut une transition plus durable (Déclaration des peuples 2009 : 8-9). Klimaforum09 apparaît alors comme un contre-mouvement à celui de marchandisation de produits fictifs.

Les attentes internationales étant très élevées lors de la conférence de Copenhague en 2009, plusieurs groupes ont profités de l'occasion pour faire valoir leur position sur la marchandisation du carbone. (CCNUCC 2014d). Le groupe World Rainforest Movement (WRM), qui « lutte contre le déboisement et l'accaparement de terres dans les pays où il existe des forêts et des communautés qui en dépendent " (WRM 2015a), a publié en vue de ces négociations sa position très critique à l'égard de cette marchandisation. L'organisation accuse le commerce du carbone d'avoir mené à un " colonialisme du climat » (WRM 2009). Cette critique fait écho aux accusations de néocolonialisme de Bachram en regard des marchés du carbone. Le WRM conteste aussi, dans une conception polanyienne, la marchandisation du carbone (WRM 2015b), mais aussi les possibilités de spéculation financière attirant les investisseurs internationaux;

" [t]he multimillionaire investor, George Soros spelt it out very clearly: "it is possible to speculate with the system: that is why it appeals to financial guys like such as myself - because that is where financial opportunities are to be found" " (WRM 2009).

D'autre part, le mouvement international La Via Campesina regroupant « des millions de paysannes et de paysans, de petits et de moyens producteurs, de sans terre, de femmes et de jeunes du monde rural, d'indigènes, de migrants et de travailleurs agricoles » milite contre le néolibéralisme et pour le climat par " la souveraineté alimentaire » (La Via Campesina 2011). Cette importante organisation participe en tant qu'ONG reconnue aux différentes $C d P$ et a publié sa position sur le commerce du carbone en prévision de la $21^{e} \mathrm{CdP}$ à Paris. L'organisation y dénonce la privatisation de la nature et l'expansion du capitalisme parce qu'elles menacent la subsistance des groupes qu'elle défend (La Via Campesina 2015). La Via Campesina peut être vue comme un groupe mariant les trois cadres théoriques présentés, car l'organisation, qui milite en partie contre le néocolonialisme, dénonce l'expansion du marché tout en attaquant le néolibéralisme comme mouvement hégémonique.

Le dernier groupe présenté est une ONG environnementale appelé Friends of Earth (FOE) ou Les Amis de la Terre, qui affirme être le plus grand réseau écologiste au monde, avec ses 73 groupes nationaux, 5000 groupes de militants locaux et plus de 2 millions de membres et sympathisants à travers le monde (FOE 
2015a). Si le groupe est majoritairement un groupe de défense de l'environnement, il rejoint plusieurs des autres causes comme la lutte contre " le néolibéralisme, la mondialisation au profit des entreprises, le néocolonialisme et le militarisme " (FOE 2015b). FOE attaque de façon virulente le commerce du carbone et les solutions contre les changements climatiques basées sur le marché (FOE 2011 : 27) et présente ces options comme " un prétexte à l'inaction désastreuse des pays développés pour réduire leurs émissions de gaz à effet de serre » (FOE 2015c : 12). L'organisation ajoute que ces mesures ne font qu'enrichir les spéculateurs désirant des gains en capital au lieu de diminuer les émissions de GES tel que promis (FOE 2009 : 4). FOE, tout comme La Via Campesina, rejoint et défend les trois cadres explicatifs de résistance au commerce du carbone.

Comme nous l'avons mentionné plus tôt, ce ne sont pas toutes les ONG qui s'opposent à la marchandisation du carbone. Ainsi, acceptant sa présence et sa potentialité à résoudre la crise climatique, certaines ONG ont décidé de ne pas la contester comme tel, mais plutôt de dénoncer ses failles afin de l'améliorer. Par exemple, la WWF a créé la certification gold standard qui certifie les crédits carbones couvrant des projets d'énergies renouvelables et de conservations d'énergie, excluant ainsi les projets de gaz industriels (MacKenzie 2009 : 452). Greenpeace, de son côté, est passée de critique de l'échange de carbone à son acceptation complète (Lohmann $2006: 58$ ).

Les groupes et mouvements que nous avons présentés précédemment apparaissent comme une exception parmi l'ensemble des groupes militants - principalement ou en partie - pour l'environnement. En effet, la majorité des ONG membres du plus gros regroupement d'ONG militant contre les changements climatiques, le Réseau action climat (RAC ou CAN), appuient dorénavant les marchés du carbone par pression du milieu de s'unir derrière une solution commune (Lohmann 2006 : 59). Ces groupes peuvent être conceptualisés comme les « débordements » du système, car ils contestent certains aspects du marché et réduisent par le fait même ses failles. Ils font partie intégrante du contremouvement en protégeant la société du « mécanisme autodestructeur » qu'est l'expansion du marché (Polanyi 2014[1944] : 127). Or, ces groupes ne travaillent pas à construire un nouveau bloc historique pouvant soutenir un mouvement contre-hégémonique.

\section{Théorisation des mouvements de contestation}

Les différents mouvements de contestation s'inscrivent dans des luttes à différentes échelles. Ils n'ont pas les mêmes objectifs ni les mêmes modes de résistance. Dans certains cas, les objectifs et les cibles ne sont pas non plus partagés. Or, les différents cadres théoriques permettent de catégoriser les mouvements de résistance en général et, par conséquent, de différencier les résistances entre elles. Dans le tableau suivant, nous présentons un résumé des théories en exposant leur objectif principal, leur mode de résistance et leur cible principale.

\begin{tabular}{|c|c|c|c|}
\hline Cadre théorique & Objectif principal & Mode de résistance & Cible principale \\
\hline Néocolonialisme & $\begin{array}{c}\text { Contrer le } \\
\text { néocolonialisme }\end{array}$ & Anticolonialisme & Les États colonisateurs \\
\hline Néogramscisme & $\begin{array}{c}\text { Créer un nouveau bloc } \\
\text { historique }\end{array}$ & Contre-hégémonie & L'appareil étatique \\
\hline Polanyisme & $\begin{array}{c}\text { Freiner l'expansion du } \\
\text { marché }\end{array}$ & Contre-mouvement & Les forces du marché \\
\hline
\end{tabular}


Dans la section précédente, nous avons pu constater les différences parfois importantes entre les mouvements de résistance à la marchandisation du carbone ${ }^{1}$. II existe parfois des similarités entre ces organisations et, tout particulièrement, dans le cadre de cette recherche, les groupes présentés avaient comme intérêts communs la défense de l'environnement et la lutte contre les changements climatiques. Dans la prochaine figure, nous présentons les mouvements de résistance dans un diagramme de Venn contenant trois cercles représentant chacun une des théories utilisées.

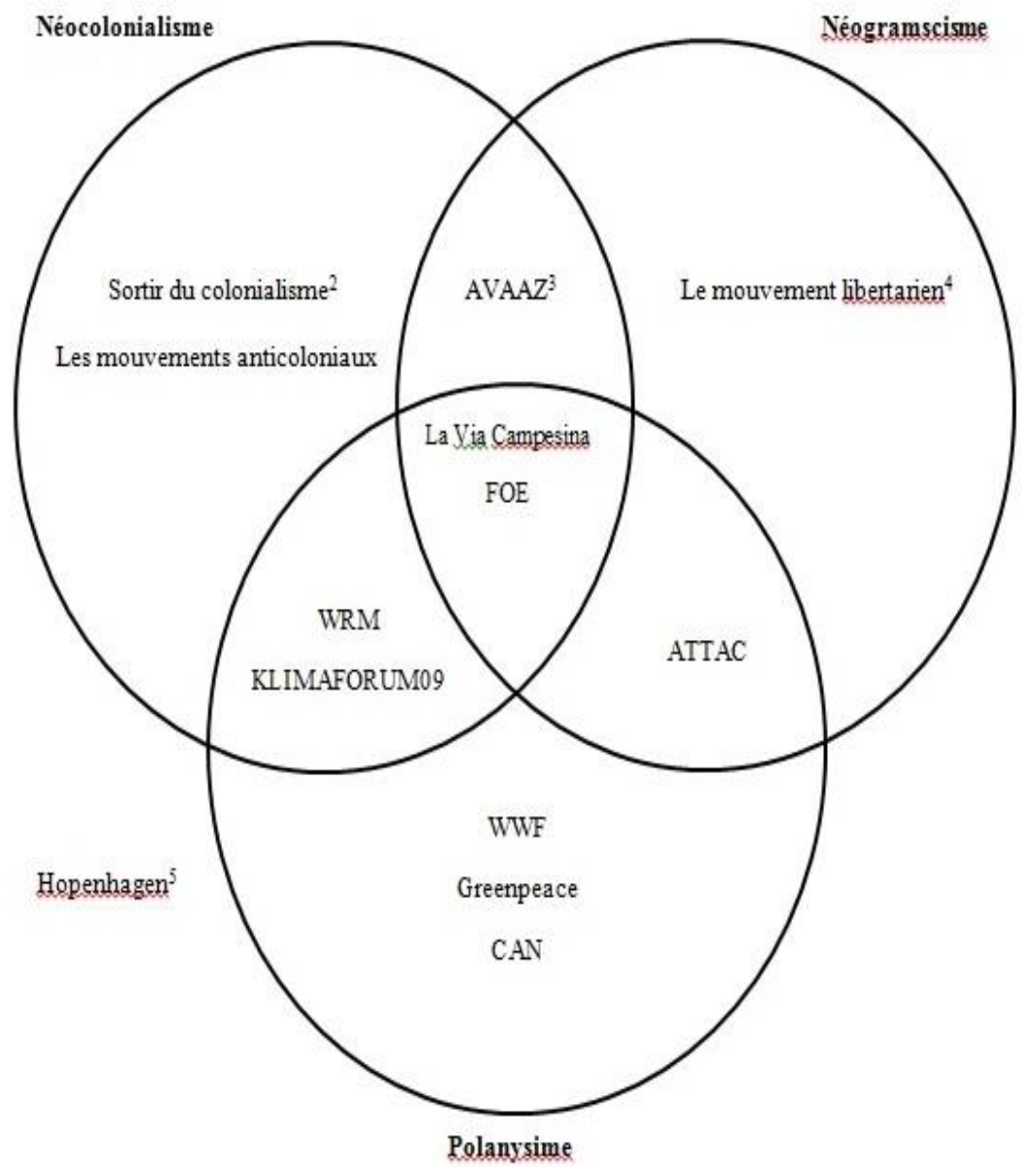

${ }^{1}$ Inspiration de Mittleman et Chin (1997) : 34.

${ }^{2}$ Sortir du colonialisme est un réseau qui « fédère des membres individuels et des organisations (associatives, politiques et syndicales), cimentés par une Charte d'objectifs communs pour sortir du colonialisme " (Sortir du colonialisme 2011). Nous n'avons pas traité de cet organisme, car il ne se positionne pas sur la marchandisation du carbone, mais nous l'utilisons comme exemple dans le tableau.

${ }^{3}$ AVAAZ est une ONG international défendant la justice climatique (Bedall $2013: 212$ ).

${ }^{4} \|$ est très difficile de trouver une organisation contre-hégémonique qui ne serait pas contre une expansion du marché. L'exemple le plus évident est le mouvement libertarien qui milite pour la quasi-disparition de l'État, voire de son élimination complète, mais dans un système où le marché domine les relations économiques (voir Murray N. Rothbard (1973), For a New Liberty - The Libertarian Manifesto, disponible en ligne, https://mises.org/library/new-liberty-libertarian-manifesto, (page consultée le 13 décembre 2015).

${ }^{5}$ Hopenhagen est une organisation défendant une économie verte, voire même le néolibéralisme vert. Hopenhagen est un exemple d'organisation qui ne peut intégrer le modèle, car elle est en faveur de l'intervention du marché pour réguler les changements climatiques (Bedall $2013: 212$ ). 
Ce diagramme nous permet de schématiser les similarités conceptuelles entre les organisations, mais aussi de voir que certaines d'entre elles se retrouvent à l'extérieur du diagramme, comme Hopenhagen, parce qu'elles ne contestent pas la marchandisation du carbone. Nous avons aussi ajouté des mouvements tels que AVAAZ, le mouvement libertarien ou le mouvement anticolonial, car ils représentent un positionnement particulier dans le diagramme. Nous avons dû, bien sûr, faire une certaine généralisation et simplification des revendications des mouvements inclus dans le diagramme pour mettre en lumière leur positionnement conceptuel. II devient alors possible de saisir la pertinence des théories présentées dans la conceptualisation de la résistance à la marchandisation du carbone.

\section{Alternatives à la marchandisation du carbone}

L'étude et la synthèse de nos trois théories nous auront permis de situer les objectifs de certains groupes environnementaux présentés dans cette recherche. Que les projets soient anticoloniaux, contrehégémoniques ou parties d'un contre-mouvement, les solutions divergent sur plusieurs aspects. Certains groupes comme la WWF, CAN ou Greenpeace militent pour la régulation des marchés du carbone et une limitation de ses dérives, alors que d'autres défendent plutôt leur abolition.

Le Klimaforum09 propose, par exemple, « une utilisation sécurisée, propre, renouvelable et durable des ressources naturelles, ainsi qu'une transition vers une souveraineté alimentaire et énergétique, foncière et sur les ressources hydriques" (Déclaration des peuples 2009). Alors que La Via Campesina propose " [I]a souveraineté alimentaire [...] comme l'une des réponses les plus crédibles aux crises actuelles de l'alimentation, de la pauvreté et du climat (La Via Campesina 2011), la FOE défend une " transition vers un système énergétique juste, durable et sans répercussions pour le climat " (FOE 2015: 14). L'organisation Carbon Trade Watch, qui dénonce ardemment la marchandisation de la nature, propose plutôt d'écouter les populations locales et d'encourager leurs projets de défense des territoires, de la biodiversité, des forêts, des lacs, des rivières et des écosystèmes interconnectés, car elles ont coexisté pendant des siècles avec ceux-ci (CTW 2014 : 13).

De son côté, Michael Löwy propose un projet de société alliant anticolonialisme, contre-hégémonie et contre-mouvement. II s'agit de l'écosocialisme. Löwy prétend que les logiques du marché sont incompatibles avec les défis environnementaux actuels (Löwy 2002 : 37). Son argumentaire se base sur deux fondements : (1) la destruction de l'environnement proviendrait de notre système de production et d'accumulation infinie provenant de la globalisation néolibérale et (2) les inégalités découlant de ce système menacerait la survivance de l'espèce humaine (Löwy 2002 : 37-38). Dans cet ordre d'idées, Löwy critique l'écologie de marché (Löwy 2008 :165), critiquant par le fait même plusieurs des organisations étudiées dans cette recherche qui étaient soit en faveur du marché du carbone, soit de sa régulation. L'écosocialisme se veut un « programme radical » contre-hégémonique tel que défini par Gramsci (Löwy 2008 : 179). Löwy propose donc un projet émancipatoire soutenant possiblement un nouveau bloc historique.

\section{Conclusion}

La présente analyse nous a permis de prendre conscience de l'importance ainsi que l'intérêt de la théorisation des mouvements de contestation et de résistance en général, mais surtout, de la résistance aux nouveaux instruments développés par le néolibéralisme pour étendre les limites du marché. La marchandisation du carbone et son commerce apparaissent effectivement comme un projet néolibéral que certains nomment le " néolibéralisme climatique » ou " néolibéralisme vert ». Ainsi, la majorité des 
mouvements de contestation du modèle néolibéral contre-hégémonique s'opposent, par extension, aux commerces du carbone. D'un autre côté, le concept de " produits fictifs » et son intégration au marché nous permettent de situer l'opposition perçue comme contre-mouvement qui propose un frein à l'expansion du marché ou du moins sa régulation étatique. En dernier lieu, plusieurs critiques des marchés du carbone proviennent de ses dérives néocoloniales et de son prolongement dans les pays du Sud. Le marché du carbone se retrouve donc accusé d'être à la fois néolibéral, néocolonial et de marchandiser la nature.

Suite à notre analyse, plusieurs questions restent en suspens. Si le marché du carbone apparaît comme la "meilleure " solution dans une économie d'inspiration néolibérale, permettra-t-elle de freiner réellement les émissions de $\mathrm{CO}_{2}$ ? À la lumière de ses failles et problèmes, il est possible d'en douter. Les créateurs et promoteurs des marchés du carbone doivent absolument palier à leurs faiblesses pour augmenter leur acceptabilité sociale. Aussi, est-ce que les marchés du carbone et leur internationalisation continueront d'être taxés de projet néocolonial en accroissant l'expropriation de terres aux communautés locales des pays en développement? Le soutien des gouvernements de ces pays en dépend assurément et par conséquent l'avenir des marchés du carbone.

En terminant, grâce aux théories présentées, nous réalisons que la résistance, dans la conception polanyienne, permet d'améliorer et de corriger les failles des marchés du carbone, tandis que les opposants au commerce du carbone doivent développer un nouveau bloc historique constitué d'alternatives tangibles permettant de réduire les émissions de carbone drastiquement et autrement. Le projet écosocialiste de Löwy apparaît prometteur dans ses capacités à réunir la contestation aux marchés du carbone et à promouvoir des solutions aux problèmes environnementaux du $21^{\mathrm{e}}$ siècle. 


\section{Bibliographie}

Abbas, Mehdi. Économie politique globale des changements climatiques. Grenoble : Presses universitaires de Grenoble, 2010.

Attac - France. «Pour une justice écologique, libérons le climat des marchés financiers ». Dernière modification le 30 novembre 2009. Page consultée le 18 octobre 2015. https://france.attac.org/nos-publications/brochures/article/pour-une-justice-ecologique.

Attac - France. 2015. «Qui sommes-nous? - Un mouvement altermondialiste ». 2015. Page consultée le 12 décembre 2015. https://france.attac.org/attac/qui-sommes-nous.

Attac - Québec. « Qui sommes-nous ? ». 2015. Page consultée le 12 décembre 2015. http://www.quebec.attac.org/?qui-sommes-nous.

Bachram, Heidi. « Climate Fraud and Carbon Colonialism: The New Trade in greenhouse Gases ». Capitalism Nature Socialism 15, no. 4 (2004): 1-16.

Baldwin, Robert. « Regulation lite: the rise of emissions trading ». Law and Financial Markets Review 2, no. 3 (2008): 262-278.

Bedall, Philip T. " 'Climate Justice'. 'Green Economy' or 'a one planet lifestyle' -Hegemonic Narratives in Transnational NGOs and Social Movements ». Dans Interpretive Approaches to Global Climate Governance -(De)constructing the Greenhouse, édité par Chris Methmann, Delf Rothe et Benjamin Stephan, 198-216. London: Routledge, 2013.

CTW. «A Tree for a Fish: The (il)logic behind selling biodiversity ». Dernière modification le 11 décembre 2014. Page consultée le 18 octobre 2015. http://www.carbontradewatch.org/articles/a-tree-fora-fish-the-il-logic-behind-selling-biodiversity.html. Carbon Trade Watch.

CCNUCC. « Historique ». 2014a. Page consultée le 2 décembre 2015. http://unfccc.int/portal_francophone/historique/items/3293.php

CCNUCC. « Le Protocole de Kyoto ». 2014b. Page consultée le 2 décembre 2015. http://unfccc.int/portal_francophone/essential_background/kyoto_protocol/items/3274.php.

CCNUCC. « Le Mécanisme de Développement Propre ». 2014c.. Page consultée le 13 décembre 2015. http://unfccc.int/portal_francophone/essential_background/feeling_the_heat/items/3297.php.

CCNUCC. «Copenhagen Climate Change Conference - December 2009 ». 2014d. Page consultée le 13 décembre 2015..http://unfccc.int/meetings/copenhagen_dec_2009/meeting/6295.php.

Cox, Robert W. "Gramsci, hegemony and international relations: An essay in method ». Dans The Global Resistance Reader, édité par Louise Amoore, 35-47. London: Routledge, 2005[1993].

Déclaration Des Peuples. « Changeons le Système ! Pas le Climat ! ». Klimaforum09. Copenhague, 2009. Page consultée le 12 décembre 2015.http://klimaforum.org/declaration_french.pdf.

De Lucia, Vito. « Hegemony and Climate Justice: A Critical Analysis ». Dans Upsetting the Offset: The Political Economy of Carbon Markets, édité par Steffen Böhm et Siddhartha Dhabi, 230-243. London: MayFlyBooks, 2009.

FOE - États-Unis. «Subprime carbon? Re-thinking the world's largest new derivatives market ». Dernière modification en mars 2009. Page consultée le 29 novembre 2015. http://www.foe.org/system/storage/877/77/4/452/SubprimeCarbonReport.pdf. 
FOE - Australie. « Contesting the Climate: Movement. Politics. Justice ». The National magazine of Friends of the Earth - Australia. Issue 111. Mars 2011.

FOE - Internationale. «À propos de nous ». 2015a. Page consultée le 13 décembre 2015. http://www.foei.org/fr/a-propos-de-nous.

FOE - Internationale. « Mission et vision ». 2015b. Page consultée le 13 décembre 2015. http://www.foei.org/fr/a-propos-de-nous/mission-et-vision.

FOE - Internationale. « Ne bradons pas le climat ». 2015c. Dernière modification le 26 novembre 2015. Page consultée le 29 novembre 2015. http://www.foei.org/fr/ressources/publicationsfr/publications-par-sujet/justice-climatique-lenergie-publications/ne-bradons-pas-le-climat.

GIEC. « Bilan 2007 des changements climatiques : Rapport de synthèse » dans Changements climatiques 2007. Groupe d'experts intergouvernemental sur l'évolution du climat. 2007.

Gilbertson, Tamra et Oscar Reyes. "Carbon Trading - How it Works and Why It Fails ». Critical Currents 7, novembre $2009: 1-102$.

Klimaforum09. «A People's Declaration from Klimaforum09 ». Klimaforum09. Copenhague. 2009. Page consultée le 12 décembre 2015. http://klimaforum.org/.

Lane, Richard et Benjamin Stephan. « Zombie markets or zombie analyses? Revivifying the politics of carbon markets ». Dans The Politics of Carbon Markets, édité par Benjamin Stephan and Richard Lane: 1-23. London: Routledge, 2015.

La Via Campesina. "Organisation - La voix des paysannes et des paysans du monde ». Dernière modification le 9 février 2011. Page consultée le 13 décembre 2015http://viacampesina.org/fr/index.php/organisation-mainmenu-44.

La Via Campesina. " Peasant agriculture is a true solution to the climate crisis ». Dernière modification le 3 septembre 2015. Page consultée le 29 novembre 2015. http://www.viacampesina.org/en/index.php/actions-and-events-mainmenu-26/-climatechange-and-agrofuels-mainmenu-75/1853-peasant-agriculture-is-a-true-solution-to-the-climatecrisis.

Lohmann, Larry. " Carbon Trading: A Critical conversation on climate change, privatisation and power ». Development Dialogues 48, (septembre 2006): 1-360.

Lohmann, Larry. "Carbon trading, climate justice and the production of ignorance: ten examples ». Development 51, no. 3 (2008): 359-365.

Lohmann, Larry. « Neoliberalism and the calculable world: The rise of carbon trading ». Dans Upsetting the Offset: The Political Economy of Carbon Markets, édité par Steffen Böhm et Siddhartha Dhabi, 25-40. London: MayFlyBooks, 2009.

Lohmann, Larry. "Uncertainty Markets and Carbon Markets: Variations on Polanyian Themes ». New Political Economy 15, no. 2 (2010): 225-254.

Lohmann, Larry. " Financialization, commodification and carbon: The contradictions of neoliberal climate policy ». Socialist Register 48 (2012): 85-107.

Löwy, Michael. « De Marx à l'écosocialisme ». Écologie \& politique 1, no. 24 (2002) : 29-41. 
Löwy, Michael. «Écosocialisme et planification démocratique ». Écologie \& politique 3, no. 37 (2008): 165-180.

Mackenzie, Donald. « Making things the same: Gases, emission rights and the politics of carbon markets ». Accounting, Organizations and Society 34, no. 3 (2009): 440-455.

Matt, Elah et Chukwumerije Okereke. « A neo-Gramscian account of carbon markets: The case of the European Union emission trading scheme and the clean development mechanism " Dans The Politics of Carbon Markets, édité par Benjamin Stephan and Richard Lane: 113-132. London: Routledge, 2015.

Melucci, Alberto. "The Symbolic Challenge of Contemporary Social Movements ». Social Research 52, no. 4 (1985): 789-816.

Newell, Peter et Matthew Paterson. "The politics of the carbon economy ». Dans The Politics of Climate Change, édité par Maxwell Boykoff: 77-95. London: Routledge, 2010.

O'Meara, Dan. «La théorie néogramscienne ". Dans Théories des relations internationales : Contestations et résistances, $2^{\mathrm{e}}$ édition, édité par Alex Macleod et Dan O'Meara: 269-314. Montréal: Athéna éditions, 2010.

Page, Edward A. " The hidden costs of carbon commodification: emissions trading, political legitimacy and procedural justice ». Democratization 19, no. 5 (2012): 932-950.

Paterson, Matthew. " Resistance Makes Carbon Markets ». Dans Upsetting the Offset: The Political Economy of Carbon Markets, édité par Steffen Böhm et Siddhartha Dhabi, 244-254. London: MayFlyBooks, 2009.

Paterson, Matthew. "Legitimation and Accumulation in Climate Change Governance ". New Political Economy. 15, no. 3 (2010): 345-368.

Polanyi, Karl. La grande transformation. Paris: Tel Gallimard, 2014 [1944].

Rothbard, Murray N. For a New Liberty - The Libertarian Manifesto. 1973. Page consultée le 13 décembre 2015. https://mises.org/library/new-liberty-libertarian-manifesto.

Sortir Du Colonialisme. "Charte du réseau Sortir du Colonialisme ». Dernière modification le 13 octobre 2011. Page consultée le 13 décembre 2015. http://www.anticolonial.net/spip.php?article2207.

Stripple, Johannes et Eva Lövbrand. "Carbon market governance beyond the public-private divide » dans Global climate governance beyond 2012 : architecture, agency and adaptation, édité par Frank Biermann. Philipp Pattberg et Fariborz Zelli. Cambridge : Cambridge University Press, 2010.

WRM. World Rainforest Movement. "Uganda: Carbon sinks and Norwegian CO2lonialism ». Dernière modification en juin 2000. Page consultée le 3 décembre 2015. http://wrm.org.uy/oldsite/bulletin/35/Uganda.html.

WRM. World Rainforest Movement. "Carbon trading: flying on the trapeze without a net ". Dernière modification le 29 novembre 2009. Page consultée le 3 décembre 2015.

http://wrm.org.uy/articles-from-the-wrm-bulletin/section2/carbon-trading-flying-on-thetrapeze-without-a-net/. 
WRM. World Rainforest Movement. « A propos du WRM ». 2015a. Page consultée le 13 décembre 2015. http://wrm.org.uy/fr/a-propos-du-wrm/.

WRM. World Rainforest Movement. «Compilation d'articles du bulletin du WRM de 2015 concernant "l'économie avec la nature" ». Dernière modification 27 novembre 2015. 2015b. Page consultée le 13 décembre 2015. http://wrm.org.uy/fr/autres-informations-pertinentes/compilationdarticles-du-bulletin-du-wrm-de-2015-concernant-leconomie-avec-la-nature/.

WWF. "The Gold Standard. Optimal Carbon Offsets ». 2015. Page consultée le 2 décembre 2015. http://wwf.panda.org/what_we_do/how_we_work/businesses/climate/offsetting/gold_standard/. 